\title{
Further Results on Pair Sum Labeling of Trees
}

\author{
Raja Ponraj $^{1}$, Jeyaraj Vijaya Xavier Parthipan ${ }^{2}$ \\ ${ }^{1}$ Department of Mathematics, Sri Paramakalyani College, Alwarkurichi, India \\ ${ }^{2}$ Department of Mathematics, St.John's College, Palayamcottai, India \\ E-mail: ponrajmath@gmail.com, parthi68@rediffmail.com
}

Received August 4, 2011; revised September 3, 2011; accepted September 10, 2011

\begin{abstract}
Let $G$ be a $(p, q)$ graph. An injective map $f: V(G) \rightarrow\{ \pm 1, \pm 2, \cdots, \pm p\}$ is called a pair sum labeling if the induced edge function, $f_{e}: E(G) \rightarrow Z-\{0\}$ defined by $f_{e}(u v)=f(u)+f(v)$ is one-one and $f_{e}(E(G))$ is either of the form $\left\{ \pm k_{1}, \pm k_{2}, \cdots, \pm k_{q / 2}\right\}$ or $\left\{ \pm k_{1}, \pm k_{2}, \cdots, k_{(q-1) / 2}\right\} \cup\left\{k_{(q+1) / 2}\right\}$ according as $q$ is even or odd. A graph with a pair sum labeling is called a pair sum graph. In this paper we investigate the pair sum labeling behavior of some trees which are derived from stars and bistars. Finally, we show that all trees of order nine are pair sum graphs.
\end{abstract}

Keywords: Path, Star, Bistar, Tree

\section{Introduction}

The graphs in this paper are finite, undirected and simple. $V(G)$ and $E(G)$ will denote the vertex set and edge set of a graph $G$. The cardinality of the vertex set of a graph $G$ is called the order of $G$ and is denoted by $p$. The cardinality of its edge set is called the size of $G$ and is denoted by $q$. The concept of pair sum labeling has been introduced in[1].The Pair sum labeling behavior of some standard graphs like complete graph, cycle, path, bistar, and some more standard graphs are investigated in [1-3]. Terms not defined here are used in the sense of Harary [4]. All the trees of order $\leq 8$ are pair sum have been proved in [5]. Here we proved that all trees of order nine are pair sum. Let $x$ be any real number. Then $\lfloor x\rfloor$ stands for the largest integer less than or equal to $x$ and $\lceil x\rceil$ stands for the smallest inter greater than or equal to $x$.

\section{Pair Sum Labeling}

Definition 2.1: Let $G$ be a $(p, q)$ graph. An injective map $f: V(G) \rightarrow\{ \pm 1, \pm 2, \cdots, \pm p\}$ is called a pair sum labeling if the induced edge function, $f_{e}: E(G) \rightarrow Z-\{0\}$ defined by is one-one and $f_{e}(E(G))$ is either of the form $\left\{ \pm k_{1}, \pm k_{2}, \cdots, \pm k_{q / 2}\right\}$ or $\left\{ \pm k_{1}, \pm k_{2}, \ldots, k_{(q-1) / 2}\right\} \cup\left\{k_{(q+1) / 2}\right\}$ according as $q$ is even or odd. A graph with a pair sum labeling defined on it is called a pair sum graph.
Notation 2.1: We denote the vertex and edge sets of star $K_{1, n}$ as follows:

$$
V\left(K_{1, n}\right)=\left\{u, u_{i}: 1 \leq i \leq n\right\}
$$

and

$$
E\left(K_{1, n}\right)=\left\{u u_{i} 1 \leq i \leq n\right\} .
$$

Notation 2.2: We denote the vertex and edge sets of bistar $B_{m, n}$ as follows:

$$
V\left(B_{m, n}\right)=\left\{u, v, u_{i}, v_{i}: 1 \leq i \leq m, 1 \leq i \leq n\right\}
$$

and

$$
E\left(B_{m, n}\right)=\left\{u v, u u_{i}, v v_{j}: 1 \leq i \leq m, 1 \leq j \leq n\right\} .
$$

Theorem 2.3 [5]: All graphs of order $\leq 8$ are pair sum.

Now we derive some pair sum trees which are used for the final section.

\section{Pair Sum Labeling of Star Related Graphs}

Here we prove that some trees which are obtained from stars are pair sum.

Theorem 3.1: The trees $G_{i},(1 \leq i \leq 5)$ with vertex set and edge set given below are pair sum.

1) $V\left(G_{1}\right)=V\left(K_{1, n}\right) \cup\left\{v_{i}: 1 \leq i \leq 6\right\}$

and 


$$
E\left(G_{1}\right)=E\left(K_{1, n}\right) \cup\left\{u v_{1}, v_{1} v_{2}, v_{2} v_{3}, v_{3} v_{4}, v_{4} v_{5}, v_{5} v_{6}\right\} .
$$

Then $G_{1}$ is a pair sum graph.

2) $V\left(G_{2}\right)=V\left(K_{1, n}\right) \cup\left\{v_{i}: 1 \leq i \leq 7\right\}$

and

$$
E\left(G_{2}\right)=E\left(K_{1, n}\right) \cup\left\{u v_{6}, v_{6} v_{7}, v_{1} v_{2}, v_{2} v_{3}, v_{3} v_{4}, v_{4} v_{5}, v_{5} u\right\} .
$$

Then $G_{2}$ is a pair sum graph.

3) $V\left(G_{3}\right)=V\left(K_{1, n}\right) \cup\left\{v_{i}: 1 \leq i \leq 7\right\}$

and

$$
E\left(G_{3}\right)=E\left(K_{1, n}\right) \cup\left\{u v_{5}, v_{5} v_{6}, v_{6} v_{7}, v_{1} v_{2}, v_{2} v_{3}, v_{3} v_{4}, v_{4} u\right\} .
$$

Then $G_{3}$ is a pair sum graph.

4) $V\left(G_{4}\right)=V\left(K_{1, n}\right) \cup\left\{v_{i} w_{i}: 1 \leq i \leq 4\right\}$

and

$$
\begin{aligned}
E\left(G_{4}\right)=E\left(K_{1, n}\right) \cup & \left\{u w_{1}, w_{1} w_{2}, u w_{3}, w_{3} w_{4}, v_{1} v_{2}, v_{2} v_{3},\right. \\
& \left.v_{3} v_{4}, v_{4} u\right\} .
\end{aligned}
$$

Then $G_{4}$ is a pair sum graph.

5) $V\left(G_{5}\right)=V\left(K_{1, n}\right) \cup\left\{v_{i}, w_{i}: 1 \leq i \leq 3\right\}$

and

$$
E\left(G_{5}\right)=E\left(K_{1, n}\right) \cup\left\{u v_{1}, u v_{2}, u v_{3}, v_{1} w_{1}, v_{2} w_{2}, v_{3} w_{3}\right\} .
$$

Then $G_{5}$ is a pair sum graph.

Proof 1): Define

$$
f: V\left(G_{1}\right) \rightarrow\{ \pm 1, \pm 2, \cdots, \pm(n+7)\}
$$

by

$$
\begin{gathered}
f(u)=-1, f\left(v_{1}\right)=-7, f\left(v_{2}\right)=-5, f\left(v_{3}\right)=1, \\
f\left(v_{4}\right)=3, f\left(v_{5}\right)=5, f\left(v_{6}\right)=7 \\
f\left(u_{i}\right)=-2 i-4,1 \leq i \leq\lceil n / 2\rceil
\end{gathered}
$$

and

$$
f\left(u_{\lfloor(n+1) / 2\rfloor+i}\right)=2 i+6,1 \leq i \leq\lceil n / 2\rceil .
$$

Then $G_{1}$ is a pair sum tree. $\square$

Proof 2): Define a map

$$
f: V\left(G_{2}\right) \rightarrow\{ \pm 1, \pm 2, \cdots, \pm(n+8)\}
$$

by

$$
\begin{gathered}
f\left(v_{1}\right)=-3, f\left(v_{2}\right)=-6, f\left(v_{3}\right)=-1, \\
f\left(v_{4}\right)=-4, f\left(v_{5}\right)=1, f\left(v_{6}\right)=3, f\left(v_{7}\right)=4, \\
f(u)=2, f\left(u_{1}\right)=7, \\
f\left(u_{1+i}\right)=4+2 i, 1 \leq i \leq\lfloor n / 2\rfloor
\end{gathered}
$$

and

$$
f\left(u_{\lceil(n / 2)+i\rceil+i}\right)=-2 i-8,1 \leq i \leq\lceil(n-2) / 2\rceil .
$$

Then $G_{2}$ is a pair sum graph. $\square$

Proof 3): Define a map

$$
f: V\left(G_{3}\right) \rightarrow\{ \pm 1, \pm 2, \cdots, \pm(n+8)\}
$$

by

$$
\begin{gathered}
f\left(v_{1}\right)=-3, f\left(v_{2}\right)=-6, f\left(v_{3}\right)=-1, \\
f\left(v_{4}\right)=-4, f\left(v_{5}\right)=2, f\left(v_{6}\right)=3, f\left(v_{7}\right)=4, \\
f(u)=1, f\left(u_{1}\right)=7+i, \\
1 \leq i \leq\lceil n / 2\rceil, f\left(u_{\lceil(n / 2)\rceil+i}\right)=-i-10,1 \leq i \leq\lfloor n / 2\rfloor .
\end{gathered}
$$

Then $G_{3}$ is a pair sum graph. $\square$

Proof 4): Define a map

$$
f: V\left(G_{4}\right) \rightarrow\{ \pm 1, \pm 2, \cdots, \pm(n+9)\}
$$

by

$$
\begin{gathered}
f(u)=-1, f\left(v_{1}\right)=3, f\left(v_{2}\right)=2, f\left(v_{3}\right)=1, f\left(v_{4}\right)=-4, \\
f\left(w_{1}\right)=-5, f\left(w_{2}\right)=-6, f\left(w_{3}\right)=7, f\left(w_{4}\right)=4 .
\end{gathered}
$$

For the other vertices we define,

$$
\begin{gathered}
f\left(u_{i}\right)=-5-2 i, 1 \leq i \leq\lceil n / 2\rceil \\
f\left(u_{\lfloor(n+1) / 2\rfloor+i}\right)=7+2 i, 1 \leq i \leq\lfloor n / 2\rfloor .
\end{gathered}
$$

Obviously $f$ is a pair sum labeling. $\square$

Proof 5): Define

$$
f: V\left(G_{5}\right) \rightarrow\{ \pm 1, \pm 2, \cdots, \pm(n+7)\}
$$

by

$$
\begin{gathered}
f(u)=-1, f\left(v_{1}\right)=2, f\left(v_{2}\right)=3, f\left(v_{3}\right)=4, \\
f\left(w_{1}\right)=-3, f\left(w_{2}\right)=-5, f\left(w_{3}\right)=-7, \\
f\left(u_{i}\right)=2 i+4,1 \leq i \leq\lceil n / 2\rceil \\
f\left(u_{\lfloor(n+1)\rfloor+i}\right)=-2-2 i, 1 \leq i \leq\lfloor n / 2\rfloor .
\end{gathered}
$$

Obviously $f$ is a pair sum labeling. $\square$

Illustration 1: A pair sum labeling of the tree $G_{1}$ with $n=10$ is

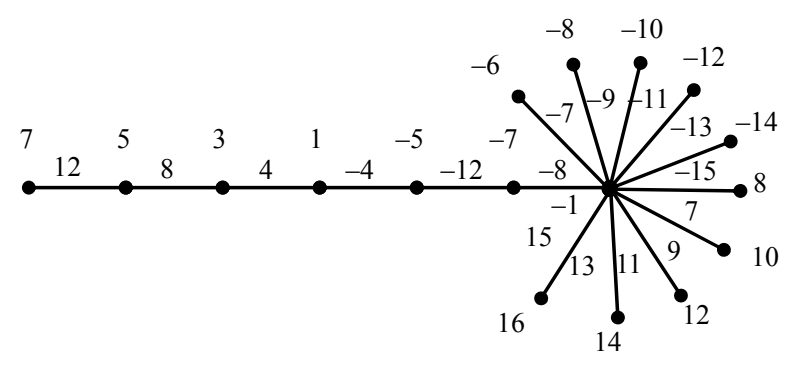


Illustration 2: A pair sum labeling of the tree $G_{3}$ with $n=9$ is

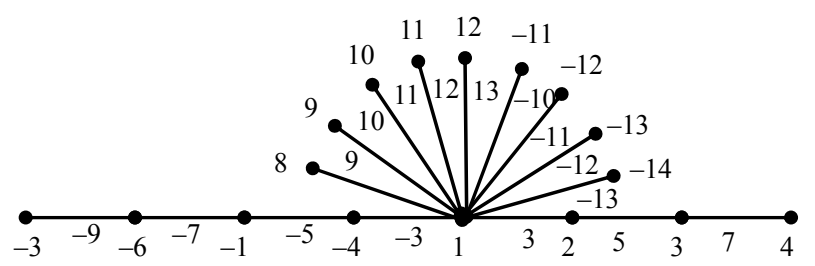

Illustration 3: A pair sum labeling of the tree $G_{5}$ with $n=11$ is

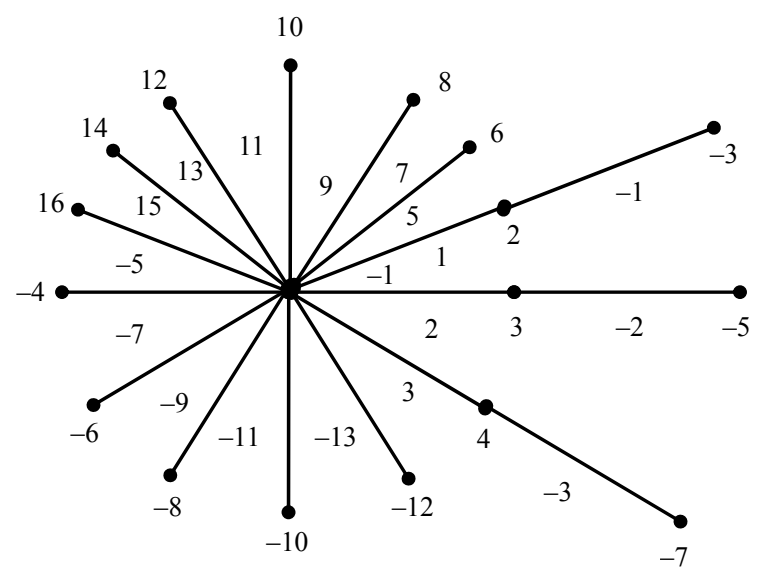

\section{Bistar Related Graphs}

In this section we show that some trees which are obtained from bistar are pair sum.

Theorem 4.1: Let $G$ be the tree with

$$
V(G)=V\left(B_{m, n}\right) \cup\left\{w_{i}: 1 \leq i \leq 6\right\}
$$

and

$$
E(G)=E\left(B_{m, n}\right) \cup\left\{v w_{1}, w_{1} w_{2}, w_{2} w_{3}, v w_{4}, w_{4} w_{5}, w_{5} w_{6}\right\} .
$$

Then $G$ is a pair sum graph.

Proof: Define

$$
f: V(G) \rightarrow\{ \pm 1, \pm 2, \cdots, \pm(m+n+8)\}
$$

and

$$
\begin{aligned}
& f\left(w_{1}\right)=4, f\left(w_{2}\right)=5, f\left(w_{3}\right)=6, f\left(w_{4}\right)=-2, \\
& f\left(w_{5}\right)=-7, f\left(w_{6}\right)=-4, f(u)=2, f(v)=-1 .
\end{aligned}
$$

Case 1): $m=n$

$$
\begin{gathered}
f\left(u_{1}\right)=-3, f\left(u_{1+i}\right)=9+i, 1 \leq i \leq n-1, \\
f\left(v_{i}\right)=-10-i, 1 \leq i \leq n .
\end{gathered}
$$

Case 2): $m>n$

Assign the label to $u_{i}, v_{i}(1 \leq i \leq n)$ as in case 1). Define

$$
f\left(u_{n+i}\right)=8+n+i, 1 \leq i \leq\lceil(m-n) / 2\rceil
$$

and

$$
f\left(u_{\lceil(m+n) / 2\rceil+i}\right)=-13-n-i, 1 \leq i \leq\lfloor(m-n) / 2\rfloor
$$

Case 3): $m<n$

Assign the label to $u_{i}, v_{i}(1 \leq i \leq m)$ as in case 1$)$. Define

$$
\begin{gathered}
f\left(v_{m+i}\right)=11+m+i, 1 \leq i \leq\lceil(n-m) / 2\rceil \\
f\left(v_{\lceil(m+n) / 2\rceil+i}\right)=-10-m-i, 1 \leq i \leq\lfloor(n-m) / 2\rfloor .
\end{gathered}
$$

Then $G$ is a pair sum graph. $\square$

Theorem 4.2: If $G$ is the tree with

$$
V(G)=V\left(B_{m, n}\right) \cup\left\{w_{i}: 1 \leq i \leq 3\right\}
$$

and

$$
E(G)=E\left(B_{m, n}\right) \cup\left\{u w_{1}, w_{1} w_{2}, w_{2} w_{3}, w_{3} v\right\} /\{u v\} .
$$

Then $G$ is a pair sum tree.

Proof: Define a function

$$
f: V(G) \rightarrow\{ \pm 1, \pm 2, \cdots, \pm(m+n+5)\}
$$

by

$$
\begin{gathered}
f(u)=-1, f(v)=3, f\left(w_{1}\right)=-4, f\left(w_{2}\right)=1, \\
f\left(w_{3}\right)=2 .
\end{gathered}
$$

Case 1): $m=n$.

$$
f\left(u_{i}\right)=-5-i, 1 \leq i \leq n
$$

and

$$
f\left(v_{i}\right)=3+i, 1 \leq i \leq n .
$$

Case 2): $m>n$.

Assign the label to $u_{i}, v_{i}(1 \leq i \leq n)$ as in case 1). Define

$$
\begin{gathered}
f\left(u_{n+i}\right)=-5-n-i, 1 \leq i \leq\lceil(m-n) / 2\rceil \\
f\left(u_{\lceil(m+n) / 2\rceil+i}\right)=7+n+i, 1 \leq i \leq\lfloor(m-n) / 2\rfloor .
\end{gathered}
$$

Then $G$ is a pair sum graph $\square$

Illustration 4: A pair sum labeling of the tree in theorem 4.2 with $m=10, n=6$ is given below: 


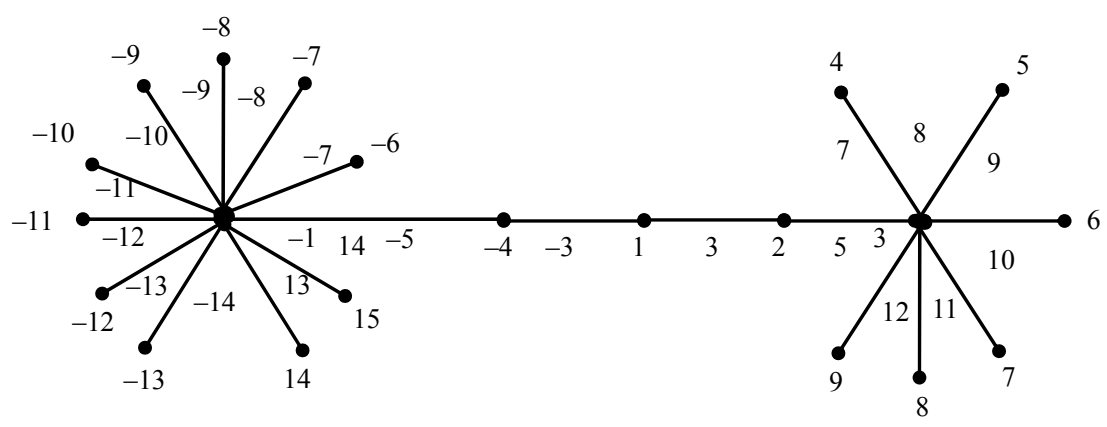

Theorem 4.3: Let $G$ be the tree with

$$
V(G)=V\left(B_{m, n}\right) \cup\left\{w_{i}: 1 \leq i \leq 4\right\}
$$

and

$$
E(G)=E\left(B_{m, n}\right) \cup\left\{u w_{1}, w_{1} w_{2}, w_{2} v, v w_{3}, w_{3} w_{4}\right\} /\{u v\} .
$$

Then $G$ is a pair sum graph.

Proof: Define

$$
f: V(G) \rightarrow\{ \pm 1, \pm 2, \cdots, \pm(m+n+6)\}
$$

by

$$
\begin{gathered}
f(u)=-1, f(v)=2, f\left(w_{1}\right)=-4, f\left(w_{2}\right)=1, \\
f\left(w_{3}\right)=3, f\left(w_{4}\right)=4 .
\end{gathered}
$$

Case 1): $m=n$.

$$
f\left(u_{1}\right)=-6, f\left(u_{i+1}\right)=-6-i, 1 \leq i \leq n-1
$$

and

$$
f\left(v_{i}\right)=5+i, 1 \leq i \leq n .
$$

Case 2): $m>n$.

Assign the label to $u_{i}, v_{i}(1 \leq i \leq n)$ as in case 1). Define

$$
f\left(u_{n+i}\right)=-5-n-i, 1 \leq i \leq\lceil(m-n) / 2\rceil
$$

and

$$
f\left(u_{\lceil(m+n) / 2\rceil+i}\right)=8+n+i, 1 \leq i \leq\lfloor(m-n) / 2\rfloor .
$$

Case 3): $m<n$.

Assign the label to $u_{i}, v_{i}(1 \leq i \leq n)$ as in case 1). Define

$$
f\left(v_{m+i}\right)=-8-m-i, 1 \leq i \leq\lceil(n-m) / 2\rceil
$$

and

$$
f\left(v_{\lceil(m+n) / 2\rceil+i}\right)=5+m+i, 1 \leq i \leq\lfloor(n-m) / 2\rfloor .
$$

Then $G$ is a pair sum graph. $\square$

Theorem 4.4: The tree $G$ with vertex set

$$
V(G)=\left(B_{m, n}\right) \cup\left\{w_{i}: 1 \leq i \leq 5\right\}
$$

and edge set

$E(G)=E\left(B_{m, n}\right) \cup\left\{u w_{1}, w_{1} v, v w_{2}, w_{2} w_{3}, v w_{4}, w_{4} w_{5}\right\} /\{u v\}$.

Then $G$ is a pair sum tree.

Proof: Define

$$
f: V(G) \rightarrow\{ \pm 1, \pm 2, \cdots, \pm(m+n+7)\}
$$

by

$$
\begin{gathered}
f(u)=-1, f(v)=1, f\left(w_{1}\right)=-4, f\left(w_{2}\right)=2, \\
f\left(w_{3}\right)=3, f\left(w_{4}\right)=-3, f\left(w_{5}\right)=5 .
\end{gathered}
$$

Case 1): $m=n$

$$
f\left(u_{i}\right)=-5-i, 1 \leq i \leq n
$$

and

$$
f\left(v_{i}\right)=5+i, 1 \leq i \leq n
$$

Case 2): $m>n$

Label the vertices $u_{i}$ and $v_{i}$ as in case 1) for $1 \leq i \leq n$. Define

$$
f\left(u_{n+i}\right)=-5-n-i, 1 \leq i \leq\lceil(m-n) / 2\rceil
$$

and

$$
f\left(u_{\lceil(m+n) / 2\rceil+i}\right)=6+n+i, 1 \leq i \leq\lceil(m-n) / 2\rceil .
$$

Case 3): $m<n$

Assign the label to $u_{i}, v_{i}(1 \leq i \leq n)$ as in case 1$)$. Define

$$
f\left(v_{m+i}\right)=5+m+i, 1 \leq i \leq\lceil(n-m) / 2\rceil
$$

and

$$
f\left(v_{\lceil(m+n) / 2\rceil+i}\right)=-7-m-i, 1 \leq i \leq\lceil(n-m) / 2\rceil .
$$

Then $G$ is a pair sum graph. $\square$

Theorem 4.5: Let $G$ be the tree with

$$
V(G)=V\left(B_{m, n}\right) \cup\left\{w_{i}: 1 \leq i \leq 5\right\}
$$

and

$E(G)=E\left(B_{m, n}\right) \cup\left\{w_{1} w_{2}, w_{2} u, u w_{3}, w_{3} v, v w_{4}, w_{4} w_{5}\right\} /\{u v\}$. 
Then $G$ is a pair sum tree.

Proof: Define a function

$$
f: V(G) \rightarrow\{ \pm 1, \pm 2, \cdots, \pm(m+n+7)\}
$$

by

$$
\begin{gathered}
f(u)=-4, f(v)=2, f\left(w_{1}\right)=-6, f\left(w_{2}\right)=-1, \\
f\left(w_{3}\right)=1, f\left(w_{4}\right)=3, f\left(w_{5}\right)=4 .
\end{gathered}
$$

Case 1): $m=n$.

$$
f\left(u_{i}\right)=-6-i, 1 \leq i \leq n
$$

and

$$
f\left(v_{i}\right)=8+i, 1 \leq i \leq n .
$$

Case 2): $m<n$.

Assign the label to $u_{i}, v_{i}(1 \leq i \leq m)$ as in case 1). Define

$$
f\left(v_{m+i}\right)=8+m+i, 1 \leq i \leq\lceil(n-m) / 2\rceil
$$

and

$$
f\left(v_{\lceil(n-m) / 2\rceil+i}\right)=-12-m-i, 1 \leq i \leq\lfloor(n-m) / 2\rfloor .
$$

Then $G$ is a pair sum graph.

Illustration 5: A pair sum labeling of the tree in theorem 4.5 with $m=6, n=11$ is given below:

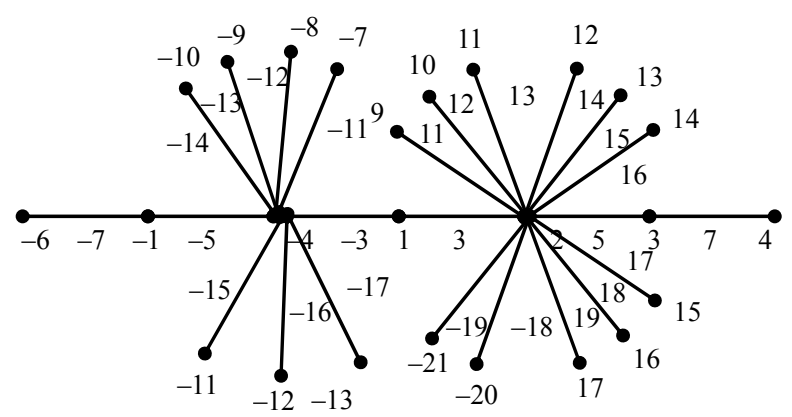

Theorem 4.6: Let $G$ be the tree with

$$
V(G)=V\left(B_{m, n}\right) \cup\left\{w_{i}: 1 \leq i \leq 4\right\}
$$

and

$$
E(G)=E\left(B_{m, n}\right) \cup\left\{u w_{1}, w_{1} v, v w_{2}, w_{2} w_{3}, w_{3} w_{4}\right\} /\{u v\} .
$$

Then $G$ is a pair sum tree.

Proof: Define a function

$$
f: V(G) \rightarrow\{ \pm 1, \pm 2, \cdots, \pm(m+n+6)\}
$$

by

$$
\begin{gathered}
f(u)=-1, f(v)=1, f\left(w_{1}\right)=-4, f\left(w_{2}\right)=2, \\
f\left(w_{3}\right)=3, f\left(w_{4}\right)=4 .
\end{gathered}
$$

Case 1): $m=n$.

$$
f\left(u_{i}\right)=-6-i, 1 \leq i \leq n
$$

and

$$
f\left(v_{i}\right)=6+i, 1 \leq i \leq n .
$$

Case 2): $m<n$.

Assign the label to $u_{i}, v_{i}(1 \leq i \leq m)$ as in case 1). Define

$$
f\left(v_{m+i}\right)=-7-m-i, 1 \leq i \leq\lceil(n-m) / 2\rceil
$$

and

$$
f\left(v_{\lceil(m+n) / 2\rceil+i}\right)=6+m+i, 1 \leq i \leq\lfloor(m-n) / 2\rfloor .
$$

Case 3): $m>n$.

Assign the label to $u_{i}, v_{i}(1 \leq i \leq n)$ as in case 1). Define

$$
f\left(u_{n+i}\right)=-5-n-i, 1 \leq i \leq\lceil(m-n) / 2\rceil
$$

and

$$
f\left(u_{\lceil(m+n) / 2\rceil+i}\right)=8+n+i, 1 \leq i \leq\lfloor(m-n) / 2\rfloor .
$$

Then $G$ is a pair sum graph. $\square$

Theorem 4.7: Let $G$ be the tree with

$$
V(G)=V\left(B_{m, n}\right) \cup\left\{w_{i}: 1 \leq i \leq 4\right\}
$$

and

$$
E(G)=E\left(B_{m, n}\right) \cup\left\{v w_{1}, w_{1} w_{2}, w_{2} w_{3}, w_{3} w_{4}\right\} .
$$

Then $G$ is a pair sum tree.

Proof: Define a function

$$
f: V(G) \rightarrow\{ \pm 1, \pm 2, \cdots, \pm(m+n+6)\}
$$

by

$$
\begin{gathered}
f(u)=1, f(v)=2, f\left(w_{1}\right)=3, f\left(w_{2}\right)=-1, \\
f\left(w_{3}\right)=-2, f\left(w_{4}\right)=-3 .
\end{gathered}
$$

Case 1): $m=n$.

$$
f\left(u_{i}\right)=-6-i, 1 \leq i \leq m
$$

and

$$
f\left(v_{1+i}\right)=3+i, 1 \leq i \leq m-1 .
$$

Case 2): $m<n$.

Assign the label to $u_{i}, v_{i}(1 \leq i \leq m)$ as in case 1). Define

$$
f\left(v_{m+i}\right)=2+m+i, 1 \leq i \leq\lceil(n-m) / 2\rceil
$$

and

$$
f\left(u_{\lceil(m+n) / 2]+i}\right)=-7-m-i, 1 \leq i \leq\lfloor(n-m) / 2\rfloor .
$$

Case 3): $m>n$. 
Assign the label to $u_{i}, v_{i}(1 \leq i \leq n)$ as in case 1$)$. Define

$$
f\left(u_{n+i}\right)=-6-n-i, 1 \leq i \leq\lceil(m-n) / 2\rceil
$$

and

$$
f\left(u_{\lceil(m+n) / 2\rceil+i}\right)=5+n+i, 1 \leq i \leq\lfloor(m-n) / 2\rfloor .
$$

Then $G$ is a pair sum graph. $\square$

Theorem 4.8: The tree $G$ with

$$
V(G)=V\left(B_{m, n}\right) \cup\left\{w_{i}: 1 \leq i \leq 5\right\}
$$

and

$$
E(G)=E\left(B_{m, n}\right) \cup\left\{v w_{1}, w_{1} w_{2}, w_{2} w_{3}, v w_{4}, w_{4} w_{5}\right\} .
$$

Then $G$ is a pair sum graph.

Proof: Define a map

$$
f: V(G) \rightarrow\{ \pm 1, \pm 2, \cdots, \pm(m+n+7)\}
$$

by

$$
\begin{gathered}
f(u)=1, f(v)=2, f\left(w_{1}\right)=3, f\left(w_{2}\right)=-4, \\
f\left(w_{3}\right)=-1, f\left(w_{4}\right)=-5, f\left(w_{5}\right)=4 .
\end{gathered}
$$

Case 1): $m=n$.

$$
f\left(u_{i}\right)=-7-i, 1 \leq i \leq m
$$

and

$$
f\left(v_{i}\right)=4+i, 1 \leq i \leq m .
$$

Case 2): $m<n$.

Assign the label to $u_{i}, v_{i}(1 \leq i \leq m)$ as in case 1$)$. Define

$$
f\left(v_{m+i}\right)=4+m+i, 1 \leq i \leq\lceil(n-m) / 2\rceil
$$

and

$$
f\left(u_{\lceil(m+n) / 2\rceil+i}\right)=-8-m-i, 1 \leq i \leq\lfloor(n-m) / 2\rfloor .
$$

Case 3): $m>n$.

Assign the label to $u_{i}, v_{i}(1 \leq i \leq n)$ as in case 1$)$. Define

$$
f\left(u_{n+i}\right)=-7-n-i, 1 \leq i \leq\lceil(m-n) / 2\rceil
$$

and

$$
f\left(u_{\lceil(m+n) / 2\rceil+i}\right)=5+n+i, 1 \leq i \leq\lfloor(m-n) / 2\rfloor .
$$

Then $G$ is a pair sum graph. $\square$

Theorem 4.9: The tree $G$ with

$$
V(G)=V\left(B_{m, n}\right) \cup\left\{w_{i}: 1 \leq i \leq 5\right\}
$$

and

$$
E(G)=E\left(B_{m, n}\right) \cup\left\{w_{1} w_{2}, w_{2} u, v w_{3}, w_{3} w_{4}, w_{4} w_{5}\right\} .
$$

Then $G$ is a pair sum graph.

Proof: Define a map

$$
f: V(G) \rightarrow\{ \pm 1, \pm 2, \cdots, \pm(m+n+7)\}
$$

by

$$
\begin{gathered}
f(u)=-4, f(v)=1, f\left(w_{1}\right)=-6, f\left(w_{2}\right)=-1, \\
f\left(w_{3}\right)=2, f\left(w_{4}\right)=3, f\left(w_{5}\right)=4 .
\end{gathered}
$$

Case 1): $m=n$.

$$
\begin{gathered}
f\left(u_{1}\right)=-5, f\left(u_{i+1}\right)=-6-i, 1 \leq i \leq m-1, \\
f\left(v_{1}\right)=8
\end{gathered}
$$

and

$$
f\left(u_{i+1}\right)=9+i, 1 \leq i \leq m-1 .
$$

Case 2): $m<n$.

Assign the label to $u_{i}, v_{i}(1 \leq i \leq m)$ as in case 1). Define

$$
f\left(v_{m+i}\right)=8+m+i, 1 \leq i \leq\lceil(n-m) / 2\rceil
$$

and

$$
f\left(u_{\lceil(m+n) / 2\rceil+i}\right)=-10-m-i, 1 \leq i \leq\lfloor(n-m) / 2\rfloor .
$$

Case 3): $m>n$.

Assign the label to $u_{i}, v_{i}(1 \leq i \leq n)$ as in case 1). Define

$$
f\left(u_{n+i}\right)=-5-n-i, 1 \leq i \leq\lceil(m-n) / 2\rceil
$$

and

$$
f\left(u_{\lceil(m+n) / 2\rceil+i}\right)=13+n+i, 1 \leq i \leq\lfloor(m-n) / 2\rfloor .
$$

Then $G$ is a pair sum graph. $\square$

Theorem 4.10: The tree $G$ with

$$
V(G)=V\left(B_{m, n}\right) \cup\left\{w_{i}: 1 \leq i \leq 6\right\}
$$

and

$$
E(G)=E\left(B_{m, n}\right) \cup\left\{w_{1} w_{2}, w_{2} u, v w_{3}, w_{3} w_{4}, v w_{5}, w_{5} w_{6}\right\} .
$$

Then $G$ is a pair sum graph.

Proof: Define a map

$$
f: V(G) \rightarrow\{ \pm 1, \pm 2, \cdots, \pm(m+n+8)\}
$$

by

$$
\begin{aligned}
& f(u)=-4, f(v)=1, f\left(w_{1}\right)=-6, f\left(w_{2}\right)=-1, \\
& f\left(w_{3}\right)=2, f\left(w_{4}\right)=3, f\left(w_{5}\right)=-3, f\left(w_{6}\right)=5 .
\end{aligned}
$$

Case 1): $m=n$.

$$
f\left(u_{i}\right)=-4-i, 1 \leq i \leq m, f\left(v_{i}\right)=6
$$

and 


$$
f\left(v_{1+i}\right)=7+i, 1 \leq i \leq m-1 .
$$

Case 2): $m<n$.

Assign the label to $u_{i}, v_{i}(1 \leq i \leq m)$ as in case 1). Define

$$
f\left(v_{m+i}\right)=6+m+i, 1 \leq i \leq\lceil(n-m) / 2\rceil
$$

and

$$
f\left(u_{\lceil(m+n) / 2\rceil+i}\right)=-8-m-i, 1 \leq i \leq\lfloor(n-m) / 2\rfloor .
$$

Case 3): $m>n$.
Assign the label to $u_{i}, v_{i}(1 \leq i \leq n)$ as in case 1). Define

$$
f\left(u_{n+i}\right)=11+n+i, 1 \leq i \leq\lceil(m-n) / 2\rceil
$$

and

$$
f\left(u_{\lceil(m+n) / 2\rceil+i}\right)=-4-n-i, 1 \leq i \leq\lfloor(m-n) / 2\rfloor .
$$

Then $G$ is a pair sum graph.

Illustration 6: A pair sum labeling of the tree in theorem 4.10 with $m=10, n=5$ is given below

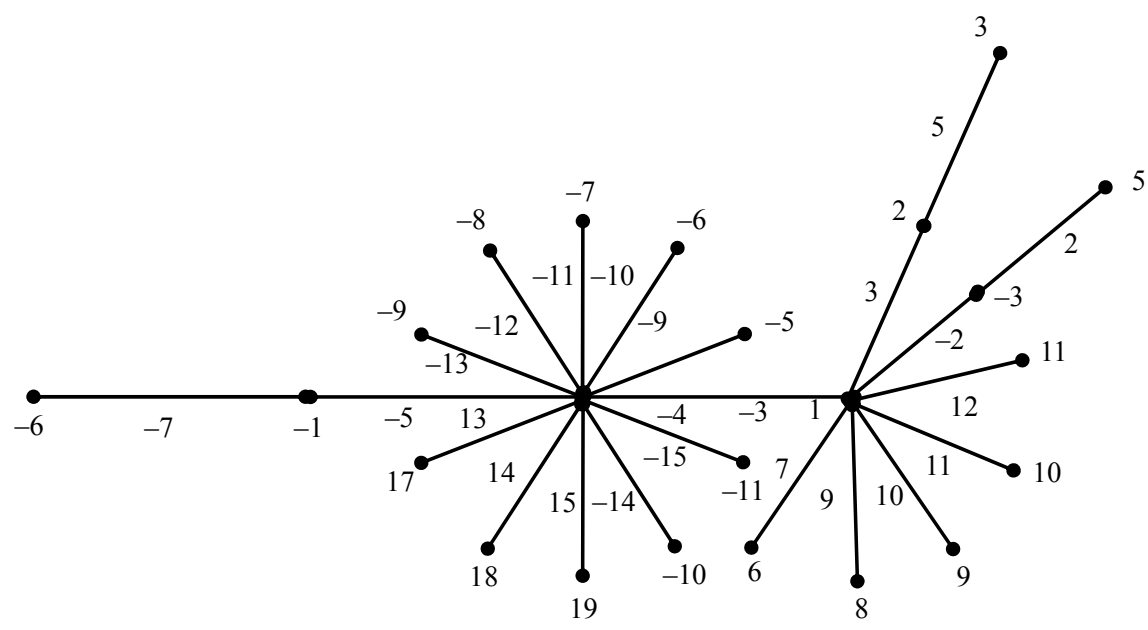

Theorem 4.11: The tree $G$ with

$$
\begin{gathered}
V(G)=V\left(B_{m, n}\right) \cup\left\{w_{i}: 1 \leq i \leq 4\right\} \\
E(G)=E\left(B_{m, n}\right) \cup\left\{w_{1} w_{2}, w_{2} u, u w_{3}, w_{3} w_{4}, w_{4} v\right\} /\{u v\} .
\end{gathered}
$$

Then $G$ is a pair sum graph.

Proof: Define a map

$$
f: V(G) \rightarrow\{ \pm 1, \pm 2, \cdots, \pm(m+n+6)\}
$$

by

$$
\begin{gathered}
f(u)=1, f(v)=-1, f\left(w_{1}\right)=3, f\left(w_{2}\right)=2, \\
f\left(w_{3}\right)=-4, f\left(w_{4}\right)=-2 .
\end{gathered}
$$

Case 1): $m=n$.

$$
f\left(u_{i}\right)=4+i, 1 \leq i \leq m
$$

and

$$
f\left(v_{i}\right)=-5-i, 1 \leq i \leq m .
$$

Case 2): $m<n$.

Assign the label to $u_{i}, v_{i}(1 \leq i \leq m)$ as in case 1). Define

$$
f\left(v_{m+i}\right)=6+m+i, 1 \leq i \leq\lceil(n-m) / 2\rceil
$$

and

$$
f\left(u_{\lceil(m+n) / 2\rceil+i}\right)=-5-m-i, 1 \leq i \leq\lfloor(n-m) / 2\rfloor .
$$

Case 3): $m>n$.

Assign the label to $u_{i}, v_{i}(1 \leq i \leq n)$ as in case 1). Define

$$
f\left(u_{n+i}\right)=4+n+i, 1 \leq i \leq\lceil(m-n) / 2\rceil
$$

and

$$
f\left(u_{\lceil(m+n) / 2\rceil+i}\right)=-6-n-i, 1 \leq i \leq\lfloor(m-n) / 2\rfloor .
$$

Then $G$ is a pair sum graph. $\square$

Theorem 4.12: The tree $G$ with

$$
V(G)=V\left(B_{m, n}\right) \cup\left\{w_{i}: 1 \leq i \leq 3\right\}
$$

$$
E(G)=E\left(B_{m, n}\right) \cup\left\{u w_{1}, w_{1} w_{2}, w_{2} w_{3}, w_{2} v\right\} /\{u v\} .
$$

Then $G$ is a pair sum graph.

Proof: Define a map

$$
f: V(G) \rightarrow\{ \pm 1, \pm 2, \cdots, \pm(m+n+5)\}
$$

by 


$$
\begin{gathered}
f(u)=-1, f(v)=1, f\left(w_{1}\right)=2, f\left(w_{2}\right)=-3, \\
f\left(w_{3}\right)=5 .
\end{gathered}
$$

Case 1): $m=n$.

$$
f\left(u_{i}\right)=-3-i, 1 \leq i \leq m
$$

and

$$
f\left(v_{i}\right)=3+i, 1 \leq i \leq m .
$$

Case 2): $m<n$.

Assign the label to $u_{i}, v_{i}(1 \leq i \leq m)$ as in case 1). Define

$$
f\left(v_{m+i}\right)=3+m+i, 1 \leq i \leq\lceil(n-m) / 2\rceil
$$

and

$$
f\left(u_{\lceil(m+n) / 2\rceil+i}\right)=-5-m-i, 1 \leq i \leq\lfloor(n-m) / 2\rfloor .
$$

Case 3): $m>n$.

Assign the label to $u_{i}, v_{i}(1 \leq i \leq n)$ as in case 1). Define

$$
f\left(u_{n+i}\right)=-3-n-i, 1 \leq i \leq\lceil(m-n) / 2\rceil
$$

and

$$
f\left(u_{\lceil(m+n) / 2\rceil+i}\right)=5+n+i, 1 \leq i \leq\lfloor(m-n) / 2\rfloor .
$$

Then $G$ is a pair sum graph. $\square$

Theorem 4.13: The tree $G$ with

$$
V(G)=V\left(B_{m, n}\right) \cup\left\{w_{i}: 1 \leq i \leq 3\right\}
$$

and

$$
E(G)=E\left(B_{m, n}\right) \cup\left\{u w_{1}, w_{1} w_{2}, w_{2} w_{3}, w_{1} v\right\} /\{u v\} .
$$

Then $G$ is a pair sum graph.

Proof: Define a map

$$
f: V(G) \rightarrow\{ \pm 1, \pm 2, \cdots, \pm(m+n+5)\}
$$

by

$$
\begin{gathered}
f(u)=-1, f(v)=3, f\left(w_{1}\right)=2, f\left(w_{2}\right)=-3, \\
f\left(w_{3}\right)=-2 .
\end{gathered}
$$

Case 1): $m=n$.

$$
\begin{gathered}
f\left(u_{i}\right)=-5-i, 1 \leq i \leq m, \\
f\left(v_{i}\right)=3+i, 1 \leq i \leq m .
\end{gathered}
$$

Case 2): $m<n$.

Assign the label to $u_{i}, v_{i}(1 \leq i \leq m)$ as in case 1). De- fine

$$
f\left(v_{m+i}\right)=3+m+i, 1 \leq i \leq\lceil(n-m) / 2\rceil
$$

and

$$
f\left(u_{\lceil(m+n) / 2]+i}\right)=-9-m-i, 1 \leq i \leq\lfloor(n-m) / 2\rfloor .
$$

Case 3): $m>n$.

Assign the label to $u_{i}, v_{i}(1 \leq i \leq n)$ as in case 1$)$. Define

$$
f\left(u_{n+i}\right)=-5-n-i, 1 \leq i \leq\lceil(m-n) / 2\rceil
$$

and

$$
f\left(u_{\lceil(m+n) / 2\rceil+i}\right)=7+n+i, 1 \leq i \leq\lfloor(m-n) / 2\rfloor .
$$

Then $G$ is a pair sum graph. $\square$

Theorem 4.14: The tree $G$ with

$$
V(G)=V\left(B_{m, n}\right) \cup\left\{w_{i}: 1 \leq i \leq 3\right\}
$$

and

$$
E(G)=E\left(B_{m, n}\right) \cup\left\{u w_{2}, w_{1} w_{2}, w_{2} w_{3}, w_{2} v\right\} /\{u v\} .
$$

Then $G$ is a pair sum graph.

Proof: Define a map

$$
f: V(G) \rightarrow\{ \pm 1, \pm 2, \cdots, \pm(m+n+5)\}
$$

by

$$
\begin{gathered}
f(u)=-1, f(v)=-3, f\left(w_{1}\right)=-5, f\left(w_{2}\right)=2, \\
f\left(w_{3}\right)=1 .
\end{gathered}
$$

Case 1): $m=n$.

$$
f\left(u_{i}\right)=4+2 i, 1 \leq i \leq m
$$

and

$$
f\left(v_{i}\right)=-2 i, 1 \leq i \leq m .
$$

Case 2): $m>n$.

Assign the label to $u_{i}, v_{i}(1 \leq i \leq n)$ as in case 1). Define

$$
f\left(u_{n+i}\right)=-3-2 n-i, 1 \leq i \leq\lceil(m-n) / 2\rceil
$$

and

$$
f\left(u_{\lceil(m+n) / 2\rceil+i}\right)=5+2 n+i, 1 \leq i \leq\lfloor(m-n) / 2\rfloor .
$$

Then $G$ is a pair sum graph.

Illustration 7: A pair sum labeling of the tree in theorem 4.14 with $m=9, n=6$ is given below: 


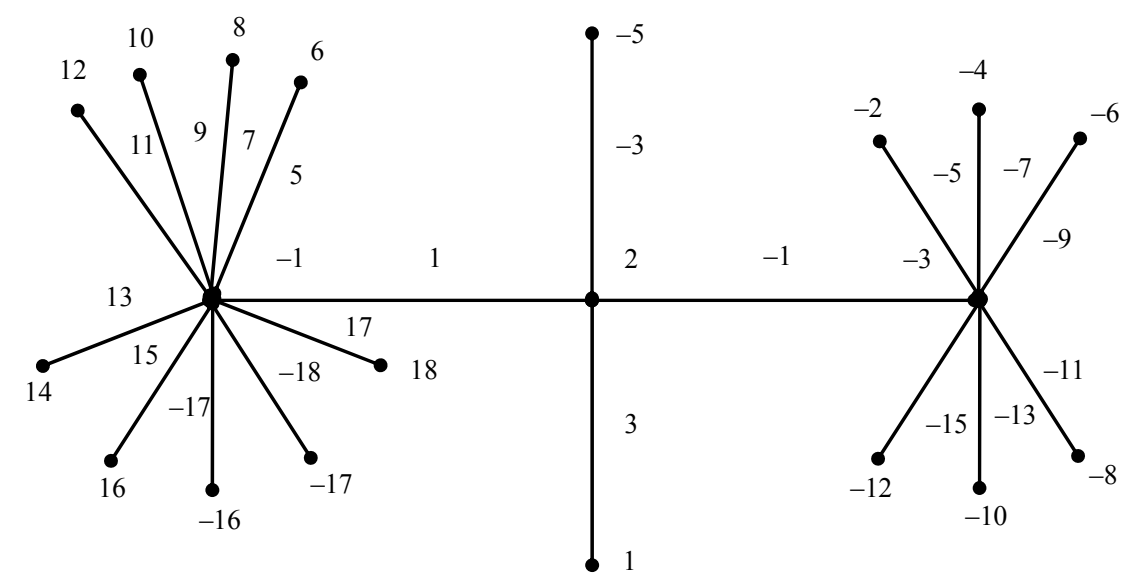

\section{Trees of Order 9}

Here we prove that all trees of order $\leq 9$ are pair sum.

Theorem 5.1: The trees given below are pair sum.
1)

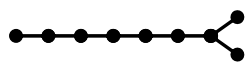

3)

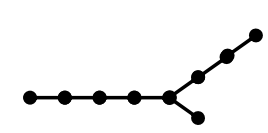

5)

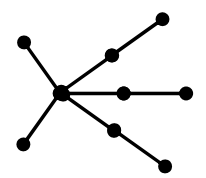

7)

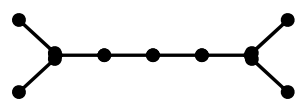

9)

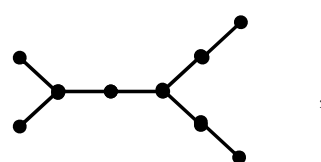

10)

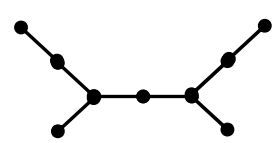

12)

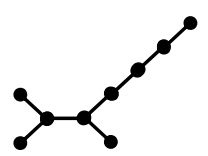

14)

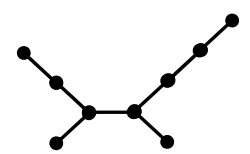

16)

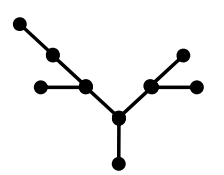

2)

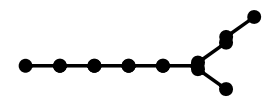

4)

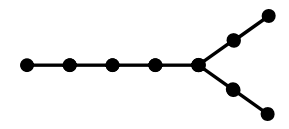

6)

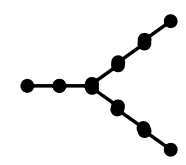

8)

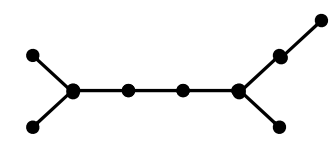

18)

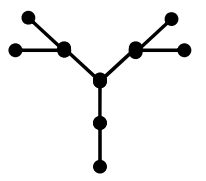

19)

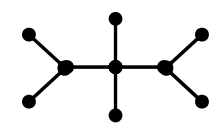

Proof: Graphs in case 1) to case 5) are pair sum by theorem 3.1. and case 6) to case 19) graphs are pair sum by theorem 4.1 to 4.14 .

Remark 5.2: The remaining trees of order 9 are pair sum by theorems in [5].

Theorem 5.3: All trees of order 9 are pair sum.

Proof: Follow from theorem 5.1 and Remark 5.2.

Theorem 5.4: All trees of order $\leq 9$ are pair sum.

Proof: Follow from theorems 2.3, 5.3. $\square$

\section{Acknowledgements}

The authors thank the referees for their comments and valuable suggestions.

\section{References}

[1] R. Ponraj and J. V. X. Parthipan, "Pair Sum Labeling of Graphs," The Journal of Indian Academy of Mathematics, Vol. 32, No. 2, 2010, pp. 587-595.

[2] R. Ponraj, J. V. X. Parthipan and R. Kala, "Some Results on Pair Sum Labeling," International Journal of Mathematical Combinatorics, Vol. 4, 2010, pp. 53-61.

[3] R. Ponraj, J. V. X. Parthipan and R. Kala, "A Note on Pair Sum Graphs," Journal of Scientific Research, Vol. 3, No. 2, 2011, pp. 321-329. doi:10.3329/jsr.v3i2.6290

[4] F. Harary, "Graph Theory," Narosa Publishing House, New Delhi, 1998.

[5] R. Ponraj, J. V. X. Parthipan and R. Kala, "Pair Sum Labeling of Some Trees," The Journal of Indian Academy of Mathematics (Communicated), in Press. 